\title{
Bounded Degree Book Embeddings and Three-Dimensional Orthogonal Graph Drawing
}

\author{
David R. Wood ${ }^{\star}$ \\ Basser Department of Computer Science \\ The University of Sydney \\ Sydney NSW 2006 \\ Australia
}

\begin{abstract}
A book embedding of a graph consists of a linear ordering of the vertices along a line in 3-space (the spine), and an assignment of edges to half-planes with the spine as boundary (the pages), so that edges assigned to the same page can be drawn on that page without crossings. Given a graph $G=(V, E)$, let $f: V \rightarrow \mathbb{N}$ be a function such that $1 \leq f(v) \leq \operatorname{deg}(v)$. We present a Las Vegas algorithm which produces a book embedding of $G$ with $O\left(\sqrt{|E| \cdot \max _{v}\lceil\operatorname{deg}(v) / f(v)\rceil}\right)$ pages, such that at most $f(v)$ edges incident to a vertex $v$ are on a single page. This algorithm generalises existing results for book embeddings. We apply this algorithm to produce 3-D orthogonal drawings with one bend per edge and $O\left(|V|^{3 / 2}|E|\right)$ volume, and single-row drawings with two bends per edge and the same volume. In the produced drawings each edge is entirely contained in some $Z$-plane; such drawings are without so-called cross-cuts, and are particularly appropriate for applications in multilayer VLSI. Using a different approach, we achieve two bends per edge with $O(|V||E|)$ volume but with cross-cuts. These results establish improved bounds for the volume of 3-D orthogonal graph drawings.
\end{abstract}

\section{Introduction}

This paper presents a Las Vegas algorithm for producing book embeddings of a graph with bounds on the number of edges incident to a vertex on a single page. This algorithm is used as the basis of algorithms for producing threedimensional orthogonal graph drawings with one and two bends per edge. We focus on drawings appropriate for applications in multilayer VLSI. Throughout this paper, $G=(V, E)$ is a undirected simple connected graph. We denote the number of vertices of $G$ by $n=|V|$, the number of edges of $G$ by $m=|E|$, and the maximum degree of $G$ by $\Delta(G)$, or $\Delta$ if the graph in question is clear.

\subsection{Book Embeddings}

A book consists of a line in 3-space, called the spine, and a number of pages, each a half-plane with the spine as boundary. A book embedding $(\pi, \rho)$ of a

\footnotetext{
* Supported by the Australian Research Council. Completed while visiting the School of Computer Science, McGill University, Canada. davidw@cs.usyd.edu.au.
} 
graph consists of a linear ordering $\pi$ of the vertices, called the spine ordering, along the spine of a book and an assignment $\rho$ of edges to pages so that edges assigned to the same page can be drawn on that page without crossings. That is, for any two edges $v w$ and $x y$, if $v<_{\pi} x<_{\pi} w<_{\pi} y$ then $\rho(v w) \neq \rho(x y)$. The book thickness of a graph $G$ is the minimum number of pages in a book embedding of $G$. For graphs with genus $\gamma$, Malitz 13 proved that the book thickness is $O(\sqrt{\gamma})$. Since $\gamma \leq m$, the book thickness is $O(\sqrt{m})$, a result proved independently by the same author [14.

Note that a book embedding may route all of the edges incident to a vertex on a single page. In this paper we study book embeddings where the number of edges incident to a vertex on a single page is bounded. We define the pagedegree of a vertex $v$ to be the maximum number of edges incident to $v$ on a single page. A bounding function of a graph $G=(V, E)$ is a function $f: V \rightarrow \mathbb{N}$ such that $1 \leq f(v) \leq \operatorname{deg}(v)$ for all vertices $v \in V$. For some bounding function $f$ of $G$, a degree- $f$ book embedding of $G$ is one in which the pagedegree of every vertex $v$ is at most $f(v)$. If for all vertices $v \in V, f(v)=c$ for some constant $c$, a degree- $f$ book embedding is simply called a degree-c book embedding.

In this paper we establish that, for an arbitrary bounding function $f$ of a graph $G$, there is a degree- $f$ book embedding with $O\left(\sqrt{m Q_{f}(G)}\right)$ pages, where $Q_{f}(G)=\max _{v \in V}\lceil\operatorname{deg}(v) / f(v)\rceil$. We describe a Las Vegas algorithm which determines this book embedding in $O\left(m \log ^{2} n \log \log m\right)$ time with high probability. This result, and its proof, generalises the above-mentioned $O(\sqrt{m})$ bound on the book thickness due to Malitz [14].

\subsection{Three-Dimensional Orthogonal Graph Drawing}

The three-dimensional orthogonal grid is the cubic lattice, consisting of gridpoints with integer coordinates, together with the axis-parallel grid-lines determined by these points. We use the word box to mean a three-dimensional axis-parallel box with integral boundaries. Boxes are possibly degenerate, in the sense that they may be rectangles, line-segments or even a single grid-point. The number of grid-points along the edge of a box parallel to the $X$-axis (respectively, $Y$-axis and $Z$-axis) is called the width (depth and height) of the box. Note that this is one more than the actual length. A $W \times D \times H$ box has width $W$, depth $D$ and height $H$. For each dimension $I \in\{X, Y, Z\}$, an $I$-line is a line parallel to the $I$-axis, an $I$-segment is a line-segment within an $I$-line, and an $I$-plane is a plane perpendicular to the $I$-axis.

A three-dimensional orthogonal drawing of a graph represents the vertices by pairwise non-intersecting boxes in the three-dimensional orthogonal grid. An edge $v w$ is represented by a sequence of contiguous segments of grid-lines possibly bent at grid-points, between the surfaces of the boxes of $v$ and $w$. The intermediate grid-points along the path representing an edge do not intersect the box of any vertex or any other edge route. From now on, we use the term drawing to mean a three-dimensional orthogonal drawing, and the graph-theoretic terms 'vertex' and 'edge' will also refer to their representation in a drawing. 
The volume of a drawing is the number of grid-points in the smallest axisaligned box, called the bounding box, which encloses the drawing. The volume and the maximum number of bends per edge are the most commonly proposed measures for determining the aesthetic quality of a drawing. A drawing with at most $b$ bends per edge is called a $b$-bend drawing. For a graph $G$, denote by $\operatorname{vol}(G, b)$ the minimum volume, taken over all $b$-bend drawings of $G$. Let $\operatorname{vol}(n, m, b)$ be the maximum, taken over all graphs $G$ with $n$ vertices and $m$ edges, of $\operatorname{vol}(G, b)$. Thus, $\operatorname{vol}(n, m, b)$ is a volume bound within which we can draw all graphs with $n$ vertices and $m$ edges, and with at most $b$ bends per edge. This paper establishes improved upper bounds on $\operatorname{vol}(n, m, 1)$ and $\operatorname{vol}(n, m, 2)$.

A drawing with height $k$ and with all vertices having height $k$ is said to be in the $k-P C B$ (Printed $C$ ircuit Board) model, as defined by Aggarwal et al. 22. Such drawings, which we call multilayer drawings, are an appropriate model for multilayer VLSI circuits. In multilayer VLSI, vertical edge segments between different $Z$-planes, called cross-cuts, lead to a deterioration in performance with an increase in the likelihood of faulty chips [2]. Therefore drawings without crosscuts are particularly desirable. In this paper we observe that for drawings with a fixed maximum number of bends per edge, permitting cross-cuts allows for drawings with less volume.

We consider three types of multilayer drawings, which are defined by the relative positions of the vertices in a $Z$-plane. A multilayer drawing has a twodimensional general position vertex layout if no two vertices are intersected by a single $X$ - or $Y$-plane. For example, the vertices may have a two-dimensional diagonal layout (see Fig. 3). We say a multilayer drawing has a linear vertex layout if every vertex is intersected by a single $X$ - or $Y$-plane (see Fig. 4). Multilayer drawings with a linear vertex layout are particularly appropriate in single-row VLSI routing problems (see 12 for example).Finally, in a grid vertex layout the vertices are positioned in a square grid (see [4]]).

1-Bend Drawings: Biedl et al. [7] construct 1-bend multilayer drawings of the complete graph $K_{n}$ with $O\left(n^{3}\right)$ volume; thus $\operatorname{vol}\left(K_{n}, 1\right) \in O\left(n^{3}\right)$ and $\operatorname{vol}(n, m, 1) \in O\left(n^{3}\right)$. Vertices are $Z$-lines of length $n$ positioned in a 2 dimensional diagonal vertex layout, and each edge is routed in some $Z$-plane. Biedl et al. 7] suggest a relationship between the assignment of $Z$-planes to edges in orthogonal drawings and the assignment of pages to edges in book embeddings. Implicit in their 1-bend drawing of $K_{n}$ is a degree-1 book embedding of $K_{n}$ with $n$ pages.

Drawings with one bend per edge were also studied by Wood [18, who shows that given a book embedding of a graph $G$ with $P$ pages, there is a 1-bend drawing of $G$ with $O(n m P)$ volume. Applying the book embedding algorithm of Malitz [13, it follows that every graph with genus $\gamma$ has a 1-bend drawing with $O(n m \sqrt{\gamma})$ volume, and since $\gamma \leq m$, it follows that $\operatorname{vol}(n, m, 1) \in O\left(n m^{3 / 2}\right)$.

The first contribution of this paper is an algorithm for producing 1bend multilayer drawings without cross-cuts and with $O\left(m n^{3 / 2}\right)$ volume; thus $\operatorname{vol}(n, m, 1) \in O\left(m n^{3 / 2}\right)$. The algorithm is based on our result for bounded de- 
gree book embeddings. Compared with the above-mentioned result of Wood [18, this represents an improvement by a factor of $\Theta\left(\sqrt{\frac{m}{n}}\right)$ for the volume of 1-bend drawings. For graphs with $m \in O\left(n^{3 / 2}\right)$ this is the best known upper bound for the volume of 1-bend drawings. (For graphs with $m \in \Omega\left(n^{3 / 2}\right)$, using the 1-bend drawing of $K_{n}$ [7] produces a drawing with less volume.)

A lower bound for the volume of 1-bend drawings was established by Biedl [3], who shows that the $\Delta$-regular $n$-vertex Ramanujan graph $G_{n, \Delta}$ has $\Omega\left(n^{2} \Delta\right)$ volume in any 1-bend drawing. Hence $\operatorname{vol}(n, m, 1) \in \Omega(n m)$. Since $K_{n}=G_{n, n-1}$, the 1-bend drawing of $K_{n}$ [7] has optimal volume; that is, $\operatorname{vol}\left(K_{n}, 1\right) \in \Theta\left(n^{3}\right)$.

2-Bend Drawings: A 2-bend multilayer drawing of $K_{n}$ in the linear layout model, with no cross-cuts and with $O\left(n^{3}\right)$ volume is presented by Biedl et al. [7]. The second contribution of this paper is an algorithm for producing 2-bend multilayer drawings with no cross-cuts and with $O\left(m n^{3 / 2}\right)$ volume. This algorithm is based on our result for bounded degree book embeddings and employs a linear vertex layout. Again, for graphs with $m \in O\left(n^{3 / 2}\right)$ this is an improved bound compared to the above result.

The algorithm of Biedl [5] produces 2-bend drawings with $O\left(n^{2} \Delta\right)$ volume in which vertices are degree-restricted; that is, the surface area of each vertex is proportional to its degree. This feature is more appropriate for applications in visualisation rather than VLSI. Strictly speaking, this algorithm does not produce multilayer drawings. However, a trivial modification does produce multilayer drawings while maintaining the volume bound, at the expense of losing the degree-restriction property. Regardless, this algorithm is not particularly appropriate for multilayer VLSI since the produced drawings have long cross-cuts.

The third contribution of this paper is an algorithm which produces 2-bend drawings with (short) cross-cuts and with $O(n m)$ volume. Hence $\operatorname{vol}(n, m, 2) \in$ $O(n m)$, which is an improvement on the above bound in [5] for all graphs. The crucial step in this algorithm is the application of an equitable edge-colouring result of Hakimi and Kariv [10].

3-Bend and 4-Bend Drawings: A 3-bend multilayer drawing of $K_{n}$ with no cross-cuts and with $O\left(n^{5 / 2}\right)$ volume is presented by Biedl et al. [7]; thus $\operatorname{vol}(n, m, 3) \in O\left(n^{5 / 2}\right)$. By placing the vertices in a $O\left(n^{1 / 2}\right) \times O\left(n^{1 / 2}\right)$ grid and routing each edge in a distinct $Z$-plane, a simple algorithm by Biedl et al. [4] produces a 3-bend multilayer drawings without cross-cuts and with $O(\mathrm{~nm})$ volume. A more complicated algorithm by the same authors produces 4-bend multilayer drawings with cross-cuts and with $O\left(m n^{1 / 2}\right)$ volume. They also prove that the Ramanujan graph $G_{n, \Delta}$ requires $\Omega\left(\Delta n^{3 / 2}\right)$ volume in any drawing. Thus $\operatorname{vol}(n, m, b) \in \Theta\left(m n^{1 / 2}\right)$ for all $b \geq 4$. Table 1 summarised the known upper bounds on the volume of drawings in the multilayer VLSI model.

The remainder of the paper is organised as follows. Our algorithm for producing degree- $f$ book embeddings is presented in Section [2. In Section [3] and Section 4 we describe our algorithms for producing 1-bend and 2-bend drawings, respectively. In Section 5 we conclude with some open problems. 
Table 1. Upper bounds on the volume of multilayer drawings.

\begin{tabular}{cccccc}
\hline \hline \# bends & bounding box & $\operatorname{vol}(n, m, b)$ & layout & cross-cuts & reference \\
\hline \hline & $O(n) \times O(n) \times O(n)$ & $O\left(n^{3}\right)$ & diagonal & no & {$[7]$} \\
1 & $O(n) \times O(n) \times O\left(m^{3 / 2}\right)$ & $O\left(n m^{3 / 2}\right)$ & diagonal & no & {$[18]$} \\
& $O(n) \times O(n) \times O\left(m n^{-1 / 2}\right)$ & $O\left(n^{3 / 2} m\right)$ & diagonal & no & Thm. [2 \\
\hline & $O(n) \times O(n) \times O(n)$ & $O\left(n^{3}\right)$ & linear & no & {$[7]$} \\
2 & $O(n) \times O(n) \times O\left(m n^{-1 / 2}\right)$ & $O\left(n^{3 / 2} m\right)$ & linear & no & Thm. [3 \\
& $O(n) \times O(n) \times O(\Delta)$ & $O\left(n^{2} \Delta\right)$ & gen. pos. & yes & {$[5]$} \\
& $O(n) \times O(n) \times O\left(m n^{-1}\right)$ & $O(n m)$ & diagonal & yes & Thm. [ [ \\
\hline 3 & $O\left(n^{1 / 2}\right) \times O\left(n^{1 / 2}\right) \times O\left(n^{3 / 2}\right)$ & $O\left(n^{5 / 2}\right)$ & grid & no & {$[7]$} \\
& $O\left(n^{1 / 2}\right) \times O\left(n^{1 / 2}\right) \times O(m)$ & $O(n m)$ & grid & no & {$[4]$} \\
\hline 4 & $O\left(n^{1 / 2}\right) \times O\left(n^{1 / 2}\right) \times O\left(m n^{-1 / 2}\right)$ & $O\left(n^{1 / 2} m\right)$ & grid & yes & {$[4]$} \\
\hline \hline
\end{tabular}

\section{Bounded Degree Book Embeddings}

This section describes a generalisation of the Las Vegas algorithm of Malitz [14 for producing degree- $f$ book embeddings. The following definitions are from [14]. A 2-coloured bipartite graph is a bipartite graph $G=\left(V_{L} \cup V_{R}, E\right)$ whose vertices have been coloured LEFT and RIGHT such that adjacent vertices are coloured differently. Note that a bipartite graph with $k$ connected components has $2^{k}$ vertex 2-colourings. For some edge $e \in E, L(e)$ refers to the end-vertex of $e$ in $V_{L}$, and $R(e)$ refers to the end-vertex of $e$ in $V_{R}$. A canonical ordering of a 2-coloured bipartite graph $G=\left(V_{L} \cup V_{R}, E\right)$ is a linear ordering of the vertices of $G$ such that all LEFT vertices precede all RIGHT vertices.

Let $\pi$ be a canonical ordering of a 2-coloured bipartite graph $G=\left(V_{L} \cup\right.$ $\left.V_{R}, E\right)$. Two edges $v w$ and $x y$ are said to cross if $v<_{\pi} x<_{\pi} w<_{\pi} y$. Two edges are disjoint if they have no common endpoint and they do not cross. Two edges intersect if they have a common endpoint or they cross. For (traditional) book embeddings the number of pairwise crossing edges provides a lower bound on the number of pages, whereas for degree-1 book embeddings the number of pairwise intersecting edges plays the same role. $G$ is completely intersecting with respect to $\pi$ if $E$ can be labelled $e_{1}, e_{2}, \ldots, e_{k}$ such that

$$
L\left(e_{1}\right) \leq_{\pi} L\left(e_{2}\right) \leq_{\pi} \cdots \leq_{\pi} L\left(e_{k}\right) \text { and } R\left(e_{1}\right) \leq_{\pi} R\left(e_{2}\right) \leq_{\pi} \cdots \leq_{\pi} R\left(e_{k}\right) .
$$

Intuitively, $G$ is completely intersecting with respect to $\pi$, if in a degree-1 book embedding with $\pi$ as the spine ordering, every edge must be placed on a unique page, as shown in Fig. 1,

Lemma 1. If a 2-coloured bipartite graph $G$ is completely intersecting with respect to some canonical ordering then $G$ is a forest.

Proof. Let $\pi$ be a canonical ordering of $G$. Suppose to the contrary that $G$ is not a forest and $G$ is completely intersecting with respect to $\pi$. Then $G$ contains 


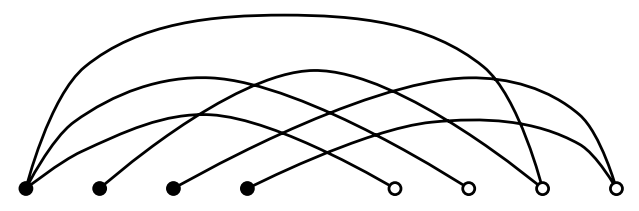

Fig. 1. A completely intersecting canonical ordering of a graph.

a cycle $\left(v_{1}, w_{1}, v_{2}, w_{2}, \ldots, v_{k}, w_{k}, v_{k+1}\right)$ with $v_{1}=v_{k+1}$ for some $k \geq 2$. Without loss of generality we can assume that $v_{1}$ is the leftmost vertex. We proceed by induction on $i$ with the following induction hypothesis: "For every $i \geq 1$, $v_{i}<_{\pi} v_{i+1}$ and $w_{i}<_{\pi} w_{i+1}$."

To prove the basis of the induction, observe that if $w_{2}<_{\pi} w_{1}$ then $v_{1} w_{1}$ does not intersect $v_{2} w_{2}$, and hence $w_{1}<_{\pi} w_{2}$. By our initial assumption, $v_{1}<_{\pi} v_{2}$. $w_{1}<_{\pi} \cdots<_{\pi} w_{i}$. If $v_{i+1}<_{\pi} v_{i}$ then $v_{i+1} w_{i}$ does not intersect $v_{i} w_{i-1}$. Thus $v_{i}<_{\pi} v_{i+1}$. If $w_{i+1}<_{\pi} w_{i}$ then $v_{i} w_{i}$ does not intersect $v_{i+1} w_{i+1}$. Thus $w_{i}<_{\pi}$ $w_{i+1}$. Therefore the inductive hypothesis holds, which is a contradiction as it implies that $v_{1}<_{\pi} v_{k+1}$ and $v_{1}=v_{k+1}$.

The next lemma for completely intersecting sets of edges, is the analogue of Lemma 2.2 in 14 for completely crossing sets of edges. Generalising a result of Tarjan [16], it says that book thickness can be determined efficiently if the spine ordering is a canonical ordering of a bipartite graph.

Lemma 2. Let $\pi$ be a canonical ordering of a 2-coloured bipartite graph $G=$ $\left(V_{L} \cup V_{R}, E\right)$ with $m$ edges and $n$ vertices. If at most $k$ edges are completely intersecting with respect to $\pi$, then a $k$-page degree-1 book embedding of $G$ with spine ordering $\pi$ can be determined in $O(m \log \log n)$ time.

Proof. Define a poset $(E, \preceq)$ as follows. For all $e_{1}, e_{2} \in E$ let

$$
e_{1} \preceq e_{2} \stackrel{\text { def }}{=} e_{1}=e_{2} \text { or }\left(L\left(e_{2}\right)<_{\pi} L\left(e_{1}\right) \text { and } R\left(e_{1}\right)<_{\pi} R\left(e_{2}\right)\right) \text {. }
$$

It is a simple exercise to check that $\leq$ is reflexive, transitive and antisymmetric, and thus is a partial order. Two edges are incomparable under $\preceq$ if and only if they intersect. Thus an antichain is a completely intersecting set of edges, and a chain is a set of pairwise disjoint edges. By Dilworth's Theorem [9] there is a decomposition of $E$ into $k$ chains where $k$ is the size of the largest antichain. That is, there is a $k$-page degree- 1 book embedding of $G$ with spine ordering $\pi$. The time complexity can be achieved using a dual form of the algorithm by Heath and Rosenberg [11, Theorem 2.3].

An equivalent result to Lemma 2 is given by Malucelli and Nicoloso [15]. To enable this lemma to be extended to degree- $f$ book embeddings, consider the following construction. Let $\pi$ be a linear ordering of the vertices of a graph $G=(V, E)$, and suppose $f$ is a bounding function of $G$. We define a graph $G_{\pi, f}$ and a linear ordering $\pi_{f}$ of $G_{\pi, f}$ as follows. Replace each vertex $v \in V$ by $f(v)$ 
consecutive vertices in $\pi_{f}$, which we call sub-vertices of $v$. As shown in Fig. 2 connect to each sub-vertex of $v$ at most $\left\lceil\frac{\operatorname{deg}(v)}{f(v)}\right\rceil$ edges incident to $v$ so that no two edges incident to a sub-vertex of $v$ cross.

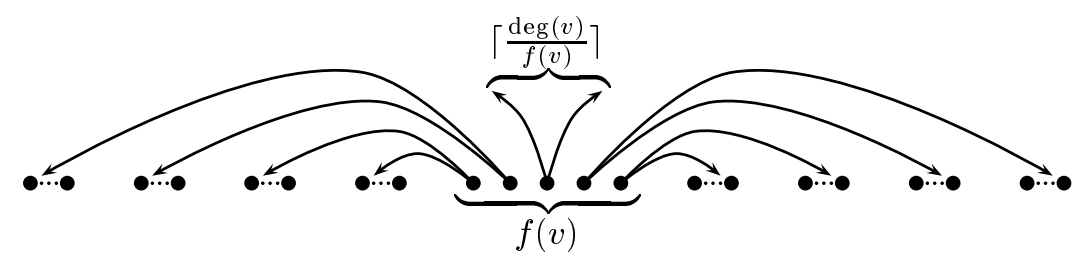

Fig. 2. Constructing $\pi_{f}$

Lemma 3. Let $f$ be a bounding function, and let $\pi$ be a canonical ordering of a 2-coloured bipartite graph $G=\left(V_{L} \cup V_{R}, E\right)$ with $m$ edges and $n$ vertices. If at most $k$ edges of $G_{\pi, f}$ are completely intersecting with respect to $\pi_{f}$, then a $k$-page degree- $f$ book embedding of $G$ with spine ordering $\pi$ can be determined in $O\left(m \log \log \left(\sum_{v} f(v)\right)\right)$ time.

Proof. Apply Lemma 2 to $G_{\pi, f}$ with spine ordering $\pi_{f}$, to obtain a degree-1 book embedding $\left(\pi_{f}, \rho\right)$ of $G_{\pi, f}$ with at most $k$ pages. In $\left(\pi_{f}, \rho\right)$, the pagedegree of a sub-vertex is at most one. Thus, in the book embedding $(\pi, \rho)$ of $G$, the pagedegree of $v$ is at most $f(v)$; that is, $(\pi, \rho)$ is a degree- $f$ book embedding of $G$. The time bound follows from Lemma 2 and that $G_{\pi, f}$ has $\sum_{v} f(v)$ vertices.

To prove the main theorem of this section, we will consider a random linear ordering of $V$. Recall that $Q_{f}(G)=\max _{v}\left\lceil\frac{\operatorname{deg}(v)}{f(v)}\right\rceil$.

Lemma 4. Let $f$ be a bounding function and let $\pi$ be a random canonical ordering of a 2-coloured forest $T=\left(V_{L} \cup V_{R}, E\right)$ with $n=\left|V_{L} \cup V_{R}\right|$ vertices. The probability that $T_{\pi, f}$ is completely intersecting with respect to $\pi_{f}$ is at most

$$
\frac{2^{n}\left(Q_{f}(T)\right)^{|E|}}{|E| !} .
$$

Proof. The probability that $T_{\pi, f}$ is completely intersecting with respect to $\pi_{f}$ is the number of canonical orderings $\pi$ of $T$ for which $T_{\pi, f}$ is completely intersecting with respect to $\pi_{f}$, divided by the number of canonical orderings of $T$. If $T_{\pi, f}$ is completely intersecting with respect to $\pi_{f}$ then all edges incident to a vertex $v$ must be incident to the same sub-vertex of $v$ in $\pi_{f}$, and thus, $T$ is completely intersecting with respect to $\pi$. (Note that this implies that $\Delta(T) \leq Q_{f}(T)$.) Thus, the desired probability is at most the number of canonical orderings $\pi$ of $T$ in which $T$ is completely intersecting, divided by the number of canonical orderings of $T$. 
We first bound the number of canonical orderings of $T$ for which $T$ is completely intersecting. Initially suppose $T$ is connected; that is, $n=|E|+1$. For some fixed ordering $\left(v_{1}, v_{2}, \ldots, v_{l}\right)$ of $V_{L}$, an ordering of $V_{R}$ which makes $T$ completely intersecting must be of the form

$$
\left\{R(e): v_{1} \in e\right\},\left\{R(e): v_{2} \in e\right\}, \ldots,\left\{R(e): v_{l} \in e\right\} .
$$

Similarly, if $\left(w_{1}, w_{2}, \ldots, w_{r}\right)$ is a fixed ordering of $V_{R}$, then an ordering of $V_{L}$ which makes $T$ completely intersecting must be of the form

$$
\left\{L(e): w_{1} \in e\right\},\left\{L(e): w_{2} \in e\right\}, \ldots,\left\{L(e): w_{l} \in e\right\} .
$$

The vertices within each set $\left\{R(e): v_{i} \in e\right\}$ and $\left\{L(e): w_{i} \in e\right\}$ possibly can be permuted. Thus the number of canonical orderings of $T$ which are completely intersecting is at most $\prod_{x} \operatorname{deg}_{T}(x)$ !.

We claim that $\prod_{x} \operatorname{deg}_{T}(x) ! \leq \Delta(T)^{|E|}$. To prove this claim, we proceed by induction on $|E|$. The basis of the induction with $|E|=1$ is trivial. Suppose for all connected trees $T^{\prime}=\left(V^{\prime}, E^{\prime}\right)$ with $\left|E^{\prime}\right|<|E|$ that $\prod_{x \in V^{\prime}} \operatorname{deg}_{T^{\prime}}(x) ! \leq \Delta\left(T^{\prime}\right)^{\left|E^{\prime}\right|}$. Let $v$ be a leaf of $T$ incident to the edge $v w$. Let $T^{\prime}=\left(V^{\prime}, E^{\prime}\right)=T \backslash\{v w\}$. Since $\operatorname{deg}_{T^{\prime}}(w)=\operatorname{deg}_{T}(w)-1$, and by the inductive hypothesis applied to $T^{\prime}$,

$$
\prod_{x \in V} \operatorname{deg}(x) !=\operatorname{deg}(w) \prod_{x \in V^{\prime}} \operatorname{deg}_{T^{\prime}}(x) \leq \operatorname{deg}(w) \cdot \Delta\left(T^{\prime}\right)^{|E|-1} \leq \Delta(T)^{|E|} .
$$

Thus the claim is proved.

Now suppose $T$ is disconnected. Then $T$ has $n-|E|$ connected components. Suppose the connected components have edge sets $E_{1}, E_{2}, \ldots, E_{n-|E|}$. For $T$ to be completely intersecting, the LEFT vertices in each connected component must be consecutive in the ordering, and similarly for the RIGHT vertices. Within $V_{L}$, the components can be ordered $(n-|E|)$ ! different ways. For a fixed ordering of the connected components of $V_{L}$, for $T$ to be completely intersecting, the components of $V_{R}$ must be ordered the same way. By (1), the number of canonical orderings which are completely intersecting is at most

$$
(n-|E|) ! \prod_{i=1}^{n-|E|} \Delta(T)^{\left|E_{i}\right|} \leq(n-|E|) ! \Delta(T)^{|E|} .
$$

The number of canonical orderings of $T$ is $\left|V_{L}\right| ! \cdot\left|V_{R}\right|$ !. Thus, the probability that a random canonical ordering of $T$ is completely intersecting is at most

$$
\frac{(n-|E|) ! \Delta(T)^{|E|}}{\left|V_{L}\right| ! \cdot\left|V_{R}\right| !} \leq \frac{(n-|E|) ! \Delta(T)^{|E|}}{\left(\frac{n}{2} !\right)^{2}} \leq \frac{2^{n}(n-|E|) ! \Delta(T)^{|E|}}{n !} \leq \frac{2^{n} \Delta(T)^{|E|}}{|E| !}
$$

where the final three inequalities follow from well-known and easily proved facts concerning factorials. The result holds, since $\Delta(T) \leq Q_{f}(T)$.

We now prove the main result of this section. It's proof is a generalisation of Theorem 2.3 in [14], which is based on ideas from Theorem 4.7 in Chung et al. [8]. 
Theorem 1. Let $f$ be a bounding function of a graph $G=(V, E)$ with $m$ edges. There exists a degree- $f$ book embedding of $G$ with $O\left(\sqrt{m Q_{f}(G)}\right)$ pages.

Proof. Let $n^{\prime}=|V|$, and denote $Q_{f}(G)$ by $Q$. Since $G$ is connected, $m \geq n^{\prime}-1$. If $m=n^{\prime}-1$ then $G$ is a tree. By considering a pre-order traversal of $G$, it is easily seen that $G$ has a book embedding $(\pi, \rho)$ with one page [8]. The graph $G_{\pi, f}$ is a forest with maximum degree $Q$, and thus has a edge-colouring $\chi$ with $Q$ colours. A book embedding $(\pi, \chi)$ of $G$ is a degree- $f$ book embedding of $G$ with $Q \leq \sqrt{\Delta Q} \leq \sqrt{m Q}$ pages. Thus the result is proved for trees.

Now assume $m \geq n^{\prime}$. Let $n=2^{\left\lceil\log n^{\prime}\right\rceil}$, and add $n-n^{\prime}$ isolated vertices to $G$. (Unless stated otherwise all logarithms are base 2.) $G$ now has $n$ vertices, with $n$ a power of 2 . Clearly, $n \leq 2 n^{\prime}$, and $n \leq 2 m$.

Let $\pi$ be a random linear ordering of $V$. For each $j, 1 \leq j \leq \log n$, divide the linear ordering $\pi$ into $2^{j}$ sections each with the same number of vertices, and label the sections from left to right $L, R, L, R$, etc. The edges whose endpoints are in adjacent $L-R$ sections (but not adjacent $R$ - $L$ sections) are called $j$-level edges. Note that every edge of $G$ appears in a unique level, and edges in adjacent $L$ - $R$ sections in some $j$-level are canonically ordered by $\pi$.

For each $j, 1 \leq j \leq \log n$, let $A_{k}^{j}$ be the event that there exists a $k$-edge 2-coloured subgraph $T$ of $G$ such that:

$-T$ consists solely of $j$-level edges,

$-T$ is canonically ordered with respect to $\pi$, and

$-T_{\pi, f}$ is completely intersecting with respect to $\pi_{f}$.

By Lemma 1 such a subgraph $T$ is a forest. The probability that $A_{k}^{j}$ occurs

$$
\mathbf{P}\left\{A_{k}^{j}\right\}<\underbrace{\left(\begin{array}{c}
m \\
k
\end{array}\right) 2^{k}} \cdot \underbrace{2^{j-1}} \cdot \underbrace{\left(\begin{array}{c}
\frac{n}{2^{j}} \\
l
\end{array}\right)\left(\begin{array}{c}
\frac{n}{2^{j}} \\
r
\end{array}\right) \frac{l ! r !(n-l-r) !}{n !}} \cdot \underbrace{\frac{2^{l+r} Q^{k}}{k !}},
$$

where:

(1) is an upper bound on the number of $k$-edge 2 -coloured forests $T$ with no isolated vertices;

(2) is the number of pairs of adjacent $L-R$ sections in the $j$-level;

(3) is an upper bound on the probability that $\pi$ canonically orders $T$ in the fixed pair of adjacent $j$-level sections, where $T$ has $l$ LEFT vertices and $r$ RIGHT vertices; and

(4) is the probability that $T$ is completely intersecting, by Lemma 4 and since $Q_{f}(T) \leq Q$.

Since $\left(\begin{array}{l}a \\ b\end{array}\right) \leq \frac{a^{b}}{b !}$,

$$
\mathbf{P}\left\{A_{k}^{j}\right\}<\frac{(2 m)^{k}}{k !} \cdot 2^{j-1} \cdot\left(\frac{n}{2^{j}}\right)^{l+r} \frac{(n-l-r) !}{n !} \cdot \frac{2^{l+r} Q^{k}}{k !} .
$$


Applying Stirling's Formula, where e is the base of the natural logarithm,

$$
\mathbf{P}\left\{A_{k}^{j}\right\}<(2 m)^{k} \cdot 2^{j-1} \cdot\left(\frac{n}{2^{j}}\right)^{l+r} \sqrt{\frac{n-l-r}{n}}\left(\frac{n-l-r}{\mathbf{e}}\right)^{n-l-r}\left(\frac{\mathbf{e}}{n}\right)^{n} \cdot \frac{2^{l+r} Q^{k} \mathbf{e}^{2 k}}{k^{2 k+1}} .
$$

Now, $n-l-r<n$. By elementary properties of a forest, $k+1 \leq l+r \leq 2 k$. Since $l+r \leq 2 \frac{n}{2^{j}}$, we have $k \leq \frac{n}{2^{j-1}}$, and hence $2^{j-1} \leq \frac{n}{k} \leq \frac{2 m}{k}$. Thus,

$$
\begin{aligned}
\mathbf{P}\left\{A_{k}^{j}\right\} & <(2 m)^{k+1} \cdot\left(\frac{1}{2^{j}}\right)^{k+1} n^{(l+r)+(n-l-r)-n} \cdot \mathbf{e}^{-(n-l-r)+n+2 k} \cdot \frac{2^{2 k} Q^{k}}{k^{2(k+1)}} \\
& <\left(\frac{8 \mathbf{e}^{4} m Q}{2^{j} k^{2}}\right)^{k+1} .
\end{aligned}
$$

Define $k_{j}=4 \mathbf{e}^{2} \sqrt{\frac{m Q}{2^{j}}}$. Since $m \geq \frac{n}{2}$ and $Q \geq 1$,

$$
\mathbf{P}\left\{A_{k_{j}}^{j}\right\}<\left(\frac{1}{2}\right)^{1+4 \mathbf{e}^{2} \sqrt{m Q / 2^{j}}}<\frac{1}{2}\left(\frac{1}{2}\right)^{2 \sqrt{2} \mathbf{e}^{2} \sqrt{n / 2^{j}}} .
$$

Consider the event that $A_{k_{j}}^{j}$ occurs for some $j, 1 \leq j \leq \log n$.

$$
\mathbf{P}\left\{\bigcup_{j=1}^{\log n} A_{k_{j}}^{j}\right\}<\frac{1}{2} \sum_{j=1}^{\log n}\left(\frac{1}{2}\right)^{2 \sqrt{2} \mathbf{e}^{2} \sqrt{n / 2^{j}}} .
$$

By induction on $N$, the following can be proved.

$$
\forall a>1, \forall b \geq \frac{\sqrt{2}-\log _{a}(a-1)}{\sqrt{2}-1}, \sum_{j=1}^{N}\left(\frac{1}{a}\right)^{b \sqrt{2^{N-j}}}<\left(\frac{1}{a}\right)^{b-1} .
$$

Applying this fact with $N=\log n, a=2$ and $b=2 \sqrt{2} \mathbf{e}^{2}$,

$$
\mathbf{P}\left\{\bigcup_{j=1}^{\log n} A_{k_{j}}^{j}\right\}<\frac{1}{2}\left(\frac{1}{2}\right)^{2 \sqrt{2} \mathbf{e}^{2}-1}=\left(\frac{1}{2}\right)^{2 \sqrt{2} \mathbf{e}^{2}}
$$

Thus,

$$
\mathbf{P}\left\{\bigcap_{j=1}^{\log n} \overline{A_{k_{j}}^{j}}\right\}=\mathbf{P}\left\{\overline{\bigcup_{j=1}^{\log n} A_{k_{j}}^{j}}\right\}=1-\mathbf{P}\left\{\bigcup_{j=1}^{\log n} A_{k_{j}}^{j}\right\}>1-\left(\frac{1}{2}\right)^{2 \sqrt{2} \mathbf{e}^{2}}>0.99999
$$

This says that for the random linear ordering $\pi$, with (very high) positive probability, $A_{k_{j}}^{j}$ does not occur for all $j, 1 \leq j \leq \log n$. Therefore, there exists a linear ordering $\pi^{\prime}$ of $V$ such that $A_{k_{j}}^{j}$ does not occur for all $j$. That is, in each pair of adjacent $L-R$ sections in the $j$-level, there is no completely intersecting subgraph in $\pi_{f}^{\prime}$ with at least $k_{j}$ edges. For each pair of adjacent $L-R$ sections in 
level $j$, apply Lemma 3 to the subgraph of $G_{\pi^{\prime}, f}$ consisting of $j$-level edges with endpoints in that pair of sections (using the canonical ordering $\pi_{f}^{\prime}$ ). By using the same set of pages for $j$-level edges, we obtain a degree- $f$ book embedding of $G$ with spine ordering $\pi^{\prime}$, and with the number of pages at most

$$
\sum_{j=1}^{\log n} k_{j}=4 \mathbf{e}^{2} \sqrt{m Q} \sum_{j=1}^{\log n} \sqrt{\frac{1}{2^{j}}}<\frac{4 \mathbf{e}^{2} \sqrt{m Q}}{\sqrt{2}-1}<72 \sqrt{m Q} .
$$

Corollary 1. Let $f$ be a bounding function of graph $G=(V, E)$ with $n$ vertices and $m$ edges. There is a Las Vegas algorithm which will compute, with high probability, a degree- $f$ book embedding of $G$ with $O\left(\sqrt{m Q_{f}(G)}\right)$ pages in $O\left(m \log ^{2} n \log \log m\right)$ time.

Proof. Consider the following Las Vegas algorithm to compute the book embedding whose existence is proved in Theorem 1 .

1. Choose a random linear ordering $\pi$ of $V$.

2. Partition the edges into $j$-levels with respect to $\pi$.

3. Embed each set of $j$-level edges in its own set of pages (using Lemma 3 . applied to $G_{\pi, f}$ as described above).

4. If the total number of pages is at most $\frac{4 \mathrm{e}^{2} \sqrt{m Q}}{\sqrt{2}-1}$ then halt. Otherwise repeat from Step 1.

The time taken for each iteration within in each $j$-level is $O\left(m \log \log \left(\sum_{v} f(v)\right)\right)$ by Lemma 3 Since $f(v) \leq \operatorname{deg}(v), \sum_{v} f(v) \in O(m)$, and the time taken for each iteration is $O(m \log n \log \log m)$. For each iteration of the above algorithm, we say the algorithm fails if the randomly chosen linear ordering $\pi$ does not admit a degree- $f$ book embedding with at most $\left(4 \mathbf{e}^{2} \sqrt{m Q}\right) /(\sqrt{2}-1)$ pages. The probability of failure is at most $2^{-2 \sqrt{2}} \mathbf{e}^{2}$. If we repeat the above algorithm at most $\log n$ times, the probability of failure every time is at most $2^{-2 \sqrt{2} \mathbf{e}^{2} \log n}=n^{-2 \sqrt{2} \mathbf{e}^{2}} \rightarrow 0$ as $n \rightarrow \infty$. Thus, with probability tending to 1 as $n \rightarrow \infty$, the above algorithm will determine a degree- $f$ book embedding of $G$ with at most $72 \sqrt{m Q_{f}(G)}$ pages in $O\left(m \log ^{2} n \log \log m\right)$ time.

Note that Theorem 1 with the bounding function $f(v)=\operatorname{deg}(v)$ is the same result proved by Malitz [14], and the above proof is based on Malitz's idea of defining $j$-levels and applying Dilworth's Theorem to a partial ordering of the edges in each level. However, our proof differs in two respects. First, we do not assume that $j \leq k$, as is the case in [14, page 76] (also see [13, page 92]). Furthermore, we do not use a book embedding of the complete graph $K_{\sqrt{n}}$ for levels $j=\frac{1}{2} \log n+1, \frac{1}{2} \log n+2, \ldots, \log n$.

\section{1-Bend Drawings}

The following simple result highlights the relationship between degree-1 book embeddings and 1-bend drawings. 
Lemma 5. If a graph $G=(V, E)$ has a degree-1 book embedding $(\pi, \rho)$ with $P$ pages then it has a 1-bend drawing with $O\left(n^{2} P\right)$ volume.

Proof. Let $\left(v_{1}, v_{2}, \ldots, v_{n}\right)$ be the numbering of vertices corresponding to the linear ordering $\pi$. Represent vertex $v_{i}$ by a $Z$-line at $(i, i)$, and draw an edge $v_{i} v_{j}$ $(i<j)$ with the route $(i, i, \rho(v w)) \rightarrow(j, i, \rho(v w)) \rightarrow(j, j, \rho(v w))$. Two edges can only intersect if they have the same $Z$-coordinate; that is they are on the same page. Such an intersection would imply a crossing in the book embedding. Hence no two edges routes intersect; see Fig. 3(a).

Given a book embedding $(\pi, \rho)$ of a graph $G=(V, E)$, consider the following elementary method for producing a degree-1 book embedding. First, apply Vizing's Theorem [17] to obtain an edge-colouring $\chi$ of $G$ with $\Delta(G)+1$ colours. (In fact a greedy edge-colouring with $O(() \Delta)$ colours will suffice.) Let $\rho^{\prime}(v w)=\rho(v w) \cdot \chi(v w)$ for all edges $v w \in E$. Then $\left(\pi, \rho^{\prime}\right)$ is a degree-1 book embedding with $O(\Delta P)$ pages. By Lemma 5 there is a 1-bend drawing with $O\left(n^{2} \Delta P\right)$ volume. This bound is reduced to $O(n m P)$ by Wood [18]. The results in [18] discussed in the introduction follow from the bounds on $P$ in [13 14.

The following algorithm for producing 1-bend drawings exploits a degree- $f$ book embedding where $f(v)$ is proportional to the ratio of the degree of $v$ and the average degree of the graph. In the produced drawing, the width and depth of a vertex $v$ equals $f(v)$. Edges in the same page are routed in a single $Z$-plane.

Theorem 2. Let $G=(V, E)$ be a graph with $n=|V|$ vertices and $m=|E|$ edges. There is a 1-bend drawing of $G$ with $O\left(m n^{3 / 2}\right)$ volume.

Proof. Let $f$ be the bounding function defined by $f(v)=\left\lceil\frac{n}{2 m} \operatorname{deg}(v)\right\rceil$ for all vertices $v \in V$. Then

$$
Q_{f}(G)=\max _{v \in V} \frac{\operatorname{deg}(v)}{\left\lceil\frac{n}{2 m} \operatorname{deg}(v)\right\rceil} \leq \frac{2 m}{n} .
$$

That is, $Q_{f}(G)$ is at most the average degree of $G$. By Theorem 1 there is degree- $f$ book embedding $(\pi, \rho)$ of $G$ with $P=O\left(\sqrt{m \frac{m}{n}}\right)=O\left(m n^{-1 / 2}\right)$ pages.

For each vertex $v$, let $S_{v}=\sum_{w<\pi} f(w)$. Represent $v$ by the $f(v) \times f(v) \times P$ box with minimum corner at $\left(S_{v}, S_{v}, 0\right)$. Clearly, vertices do not intersect. The $i$ th successor of $v$ on a page $p$ is the edge $v x_{i}$ in the list $v x_{1}, v x_{2}, \ldots, v x_{k}$ of edges incident to $v$ on page $p$ such that $v<_{\pi} x_{1}<_{\pi} x_{2}<_{\pi} \cdots<_{\pi} x_{k}$. The $i$ th predecessor of $v$ on page $p$ is the edge $v x_{i}$ in the list $v x_{1}, v x_{2}, \ldots, v x_{k}$ of edges incident to $v$ on page $p$ such that $x_{k}<_{\pi} x_{k-1}<_{\pi} \cdots<_{\pi} x_{1}<_{\pi} v$. For every edge $v w\left(v<_{\pi} w\right)$, if $v w$ is the $i$ th successor of $v$ on page $\rho(v w)$ and the $j$ th predecessor of $w$ on page $\rho(v w)$, then draw $v w$ with the 1-bend edge route $\left(S_{v}+f(v)-1, S_{v}+f(v)-i, \rho(v w)\right) \rightarrow\left(S_{w}+j-1, S_{v}+f(v)-i, \rho(v w)\right) \rightarrow$ $\left(S_{w}+j-1, S_{w}, \rho(v w)\right)$, as illustrated in Fig. 3(b).

Two edges can only intersect if they have the same $Z$-coordinate; that is they are on the same page. Such an intersection would imply a crossing in the book embedding. Hence no two edges intersect. The width and depth of the bounding box is $\sum_{v}\left(\left\lceil\frac{n}{2 m} \operatorname{deg}(v)\right\rceil+1\right) \leq 2 n+\frac{n}{2 m}(2 m)=3 n$. The height of the bounding box is $P=O\left(m n^{-1 / 2}\right)$. Thus the volume is $O\left(m n^{3 / 2}\right)$. 


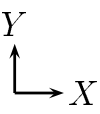

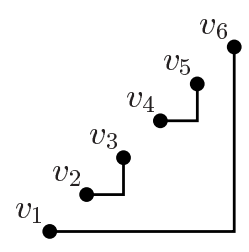

(a)

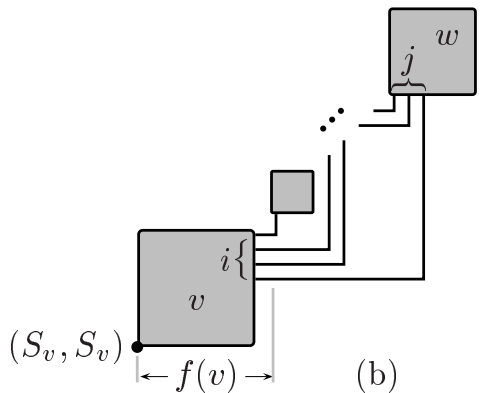

(b)

Fig. 3. 1-bend edge routes within a $Z$-plane.

\section{2-Bend Drawings}

We now present our algorithms for producing 2-bend drawings, the first with a linear vertex layout, and the second with a diagonal vertex layout.

Theorem 3. Every graph $G=(V, E)$ with $n$ vertices and $m$ edges has a 2-bend linear drawing with $O\left(m n^{3 / 2}\right)$ volume.

Proof. Let $f$ be the same bounding function defined in Theorem 2 . By Theorem 1 there is degree- $f$ book embedding $(\pi, \rho)$ of $G$ with $P=O\left(m n^{-1 / 2}\right)$ pages. For each vertex $v \in V$, let $S_{v}=\sum_{w<\tau} f(w)$. Represent $v$ by the $f(v) \times 1 \times P$ box with minimum corner at $\left(S_{v}, 0,0\right)$. Clearly, vertices do not intersect.

Denote by $\omega(v w)$, the pagewidth of an edge $v w$ in $(\pi, \rho)$; that is, the maximum number of edges cut by a line-segment contained in $\rho(v w)$, perpendicular to the spine ordering, and with endpoints on $v w$ and the spine.

For every vertex $v \in V$ and page $p$, the $i$ th neighbour of $v$ on page $p$ is the edge $v x_{i}$ in the list $v x_{1}, v x_{2}, \ldots, v x_{k}$ of edges incident to $v$ on page $p$ such that $x_{1}<_{\pi}$ $x_{2}<_{\pi} \cdots<_{\pi} x_{k}$. For every edge $v w\left(v<_{\pi} w\right)$, if $v w$ is the $i$ th neighbour of $v$ on page $\rho(v w)$, and $v w$ is the $j$ th neighbour of $w$ on page $\rho(v w)$, then draw $v w$ with the 2-bend edge route $\left(S_{v}+i-1,0, \rho(v w)\right) \rightarrow\left(S_{v}+i-1, \omega(v w), \rho(v w)\right) \rightarrow$

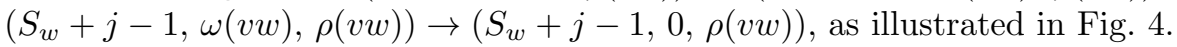

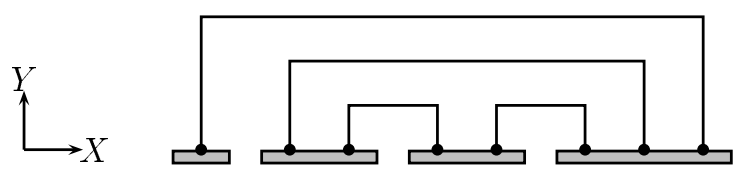

Fig. 4. A $Z$-plane within a 2-bend linear drawing

Two edges can only intersect if they have the same $Z$-coordinate; that is, they are on the same page of the book embedding. Clearly $Y$-segments do not 
intersect. An $X$-segment intersecting a $Y$-segment would imply a crossing in the book embedding. Two $X$-segments on the same page either have no $X$-coordinate in common, or they are nested, and therefore have different pagewidth, and thus have different $Y$-coordinates. Hence no two edges routes intersect.

The maximum pagewidth and hence the depth of the bounding box is at most the width of the bounding box. As in Theorem 2, the width and hence the depth of the bounding box is $O(n)$. The height of the bounding box is $P=O\left(m n^{-1 / 2}\right)$, and thus the volume is $O\left(m n^{3 / 2}\right)$.

The next drawing algorithm, which does not use a book embedding, exploits a (non-proper) edge-colouring in which the colours are evenly distributed about the edges incident to each vertex. The colour of an edge determines its 'height' in the drawing.

Theorem 4. Every graph $G=(V, E)$ with $n$ vertices and $m$ edges has a 2-bend multilayer drawing with cross-cuts and with $O(n m)$ volume.

Proof. Hakimi and Kariv [10, Theorem 3] prove that for every $k \in \mathbb{N}$, every graph has a (non-proper) edge $k$-colouring such that the number of monochromatic edges incident to a vertex $v$ is at most $\left\lceil\frac{1}{k}(\operatorname{deg}(v)+1)\right\rceil$. Apply this result with $k=\left\lceil\frac{2 m}{n}\right\rceil$, to obtain an edge $\left\lceil\frac{2 m}{n}\right\rceil$-colouring $\chi$ of $G$, such that the number of monochromatic edges incident to a vertex $v$ is at most $\left\lceil(\operatorname{deg}(v)+1) /\left\lceil\frac{2 m}{n}\right\rceil\right\rceil \leq$ $\left\lceil\frac{n}{2 m}(\operatorname{deg}(v)+1)\right\rceil$. Let $\pi$ be an arbitrary linear ordering of $V$. For each vertex $v$, let $S_{v}=\left\lceil\frac{n}{2 m}(\operatorname{deg}(v)+1)\right\rceil$ and $T_{v}=\sum_{w<_{\pi} v} S_{w}$. Represent $v$ by the $S_{v} \times S_{v} \times 2\left\lceil\frac{2 m}{n}\right\rceil$ box with minimum corner at $\left(T_{v}, T_{v}, 0\right)$. Clearly, vertices do not intersect.

Define the $i$ th successor and $i$ th predecessor of a vertex $v$ with colour $c$ as in Theorem[2] but with "colour" replacing "page". For every edge $v w\left(v<_{\pi} w\right)$, if $v w$ is the $i$ th successor of $v$ coloured $\chi(v w)$, and $v w$ is the $j$ th predecessor of $w$ coloured $\chi(v w)$, then draw $v w$ with the 2-bend edge route $\left(T_{v}+S_{v}-1\right.$, $\left.T_{v}+S_{v}-i, 2 \chi(v w)\right) \rightarrow\left(T_{w}+j-1, T_{v}+S_{v}-i, 2 \chi(v w)\right) \rightarrow\left(T_{w}+j-1, T_{v}+\right.$ $\left.S_{v}-i, 2 \chi(v w)-1\right) \rightarrow\left(T_{w}+j-1, T_{w}, 2 \chi(v w)-1\right)$.

The $i$ th successor of a vertex $v$ coloured $c$ is a unique edge, and similarly for predecessors. Thus two $X$-segments have different $Z$-coordinates or different $Y$-coordinates, and any two $Y$-segments have different $Z$-coordinates or different $X$-coordinates. Hence such edge-segments do not intersect. Since $X$ segments have even $Z$-coordinates, and $Y$-segments have odd $Z$-coordinates, no $X$-segment intersects a $Y$-segment. The unit-length $Z$-segment in each edge does not intersect any other edges as this would imply that one of the adjacent $X$ - or $Y$-segments would have been involved in an intersection. Thus no two edges intersect. The width and depth of the bounding box is $\sum_{v}\left(\left\lceil\frac{n}{2 m}(\operatorname{deg}(v)+1)\right\rceil+1\right) \leq$ $2 n+\frac{n}{2 m}(2 m+n) \leq 2 n+\frac{n}{2 m}(4 m)=4 n$. The height of the bounding box is $O\left(\frac{m}{n}\right)$. Therefore the volume is $O(\mathrm{~nm})$.

\section{Conclusion}

We have presented a Las Vegas algorithm for producing book embeddings with bounds on the pagedegree of each vertex. This algorithm is used to produce 
1-bend and 2-bend drawings without cross-cuts. Using an approach based on equitable edge-colourings we described an algorithm for producing 2-bend drawings with cross-cuts and with the best known volume upper bound. For all of our algorithms constant-factor improvements are easily possible. For example, in the 1-bend algorithm half the pages can be routed in the space with $Y$-coordinate greater than $X$-coordinate. In the 2-bend algorithm with a diagonal layout, $2-\mathrm{D}$ general position vertex layouts [6] can be used to reduce the width and depth.

We finish with some open problems. First, what are values of $\operatorname{vol}(n, m, 1)$, $\operatorname{vol}(n, m, 2)$ and $\operatorname{vol}(n, m, 3)$ ? The best known bounds are $\Omega(n m) \quad \ni$ $\operatorname{vol}(n, m, 1) \in O\left(\min \left\{m n^{3 / 2}, n^{3}\right\}\right)$, and for $b \in\{2,3\}, \Omega\left(n^{1 / 2} m\right) \ni \operatorname{vol}(n, m, b) \in$ $O(n m)$. For $b \geq 4, \operatorname{vol}(n, m, b) \in \Theta\left(m n^{1 / 2}\right)$. The algorithm in 4 produces multilayer drawings with $O\left(m n^{1 / 2}\right)$ volume but with cross-cuts. Does every graph have a multilayer drawing with no cross-cuts and with $O\left(m n^{1 / 2}\right)$ volume? Some progress towards a positive answer to this question is presented in [19]. If the answer is negative, is there a lower bound of $\Omega(\mathrm{nm})$ for the volume of multilayer drawings with no cross-cuts regardless of the number of bends?

Acknowledgements. Thanks to Therese Biedl for stimulating discussions on this topic, and to everyone in the School of Computer Science at McGill University, especially Sue Whitesides, for their generous hospitality.

\section{References}

1. Proc. EuroConference on Combinatorics, Graph Theory and Applications (COMB'01), Electronic Notes in Discrete Mathematics, to appear.

2. A. Aggarwal, M. Klawe, and P. Shor. Multilayer grid embeddings for VLSI. Algorithmica, 6(1):129-151, 1991.

3. T. Biedl. 1-bend 3-D orthogonal box-drawings: Two open problems solved. $J$. Graph Algorithms Appl., 5(3):1-15, 2001.

4. T. Biedl, T. Thiele, and D. R. Wood. Three-dimensional orthogonal graph drawing with optimal volume. In J. Marks, editor, Proc. Graph Drawing: 8th International Symp. (GD'00), volume 1984 of Lecture Notes in Comput. Sci., pages 284-295. Springer, 2001.

5. T. C. Biedl. Three approaches to 3D-orthogonal box-drawings. In S. Whitesides, editor, Proc. Graph Drawing: 6th International Symp. (GD'98), volume 1547 of Lecture Notes in Comput. Sci., pages 30-43. Springer, 1998.

6. T. C. Biedl and M. Kaufmann. Area-efficient static and incremental graph drawings. In R. Burkhard and G. Woeginger, editors, Proc. Algorithms: 5th Annual European Symp. (ESA'97), volume 1284 of Lecture Notes in Comput. Sci., pages 37-52. Springer, 1997.

7. T. C. Biedl, T. Shermer, S. Whitesides, and S. Wismath. Bounds for orthogonal 3-D graph drawing. J. Graph Algorithms Appl., 3(4):63-79, 1999.

8. F. R. K. Chung, F. T. Leighton, and A. L. Rosenberg. Embedding graphs in books: a layout problem with applications to VLSI design. SIAM J. Algebraic Discrete Methods, 8(1):33-58, 1987.

9. R. P. Dilworth. A decomposition theorem for partially ordered sets. Ann. of Math. (2), 51:161-166, 1950. 
10. S. L. Hakimi and O. Kariv. A generalization of edge-coloring in graphs. J. Graph Theory, 10(2):139-154, 1986.

11. L. S. Heath and A. L. Rosenberg. Laying out graphs using queues. SIAM J. Comput., 21(5):927-958, 1992.

12. E. S. Kuh, T. Kashiwabara, and T. Fujisawa. On optimum single-row routing. IEEE Trans. Circuits and Systems, 26(6):361-368, 1979.

13. S. M. Malitz. Genus $g$ graphs have pagenumber $O(\sqrt{g})$. J. Algorithms, 17(1):85109, 1994

14. S. M. Malitz. Graphs with $E$ edges have pagenumber $O(\sqrt{E})$. J. Algorithms, 17(1):71-84, 1994.

15. F. Malucelli and S. Nicoloso. Optimal partition of a bipartite graph into noncrossing matchings. In 1 .

16. R. Tarjan. Sorting using networks of queues and stacks. J. Assoc. Comput. Mach., 19:341-346, 1972.

17. V. G. Vizing. On an estimate of the chromatic class of a $p$-graph. Diskret. Analiz No., 3:25-30, 1964.

18. D. R. Wood. Three-dimensional orthogonal graph drawings with one bend per edge. Submitted. See Three-Dimensional Orthogonal Graph Drawing. Ph.D. thesis, School of Computer Science and Software Engineering, Monash University, Australia, 2000.

19. D. R. Wood. Geometric thickness in a grid of linear area. In 1 . 